\title{
Migdal Effect in Semiconductors
}

\author{
Simon Knapen, ${ }^{1, *}$ Jonathan Kozaczuk, ${ }^{2, \dagger}$ and Tongyan $\operatorname{Lin}^{2, \$}$ \\ ${ }^{1}$ CERN, Theoretical Physics Department, 1211 Geneva 23, Switzerland \\ ${ }^{2}$ Department of Physics, University of California, San Diego, California 92093, USA
}

(Received 2 December 2020; accepted 14 July 2021; published 20 August 2021)

\begin{abstract}
When a nucleus in an atom undergoes a collision, there is a small probability of an electron being excited inelastically as a result of the Migdal effect. In this Letter, we present the first complete derivation of the Migdal effect from dark matter-nucleus scattering in semiconductors, which also accounts for multiphonon production. The rate of the Migdal effect can be expressed in terms of the energy loss function of the material, which we calculate with density functional theory methods. Because of the smaller gap for electron excitations, we find that the rate for the Migdal effect is much higher in semiconductors than in atomic targets. Accounting for the Migdal effect in semiconductors can therefore significantly improve the sensitivity of experiments such as DAMIC, SENSEI, and SuperCDMS to sub-GeV dark matter.
\end{abstract}

DOI: 10.1103/PhysRevLett.127.081805

Introduction.-Direct detection of nuclear recoils from sub-GeV dark matter (DM) is challenging because the typical energy deposited in an elastic nuclear recoil scales as $E_{N} \sim m_{\chi}^{2} v_{\chi}^{2} / m_{N}$, which is exceedingly difficult to detect for lighter dark matter candidates. Furthermore, low-energy nuclear recoils do not deposit much energy in the form of charge (electrons) or scintillation light, which are the primary detection channels in many experiments. These considerations are drivers for new technologies and experiments capable of lower thresholds and phonon-based detection $[1,2]$.

In the meantime, existing experiments can significantly extend their reach by searching for inelastic scattering processes during which additional excitations are created, e.g., ionization electrons [3], photons [4], or plasmons $[5,6]$. While there is generally a rate penalty for such processes, there are two key advantages: (i) the unfavorable energy relation in the previous paragraph can be broken and (ii) the additional excitation gives rise to charge signals that are more readily detected.

The Migdal effect [7,8] describes inelastic collisions where atoms are excited or ionized during the initial hard nuclear recoil. For $m_{\chi} \gtrsim 70 \mathrm{MeV}$, this process takes place on timescales much shorter than the time for the recoiling nucleus to travel a distance comparable to the interatomic spacing. It can therefore be factorized from secondary ionizations that can occur as the recoiling nucleus interacts with surrounding atoms, as described by Lindhard's theory

Published by the American Physical Society under the terms of the Creative Commons Attribution 4.0 International license. Further distribution of this work must maintain attribution to the author(s) and the published article's title, journal citation, and DOI. Funded by SCOAP ${ }^{3}$.
[9]. As we will show, in crystals the underlying physics of the primary (Migdal) and secondary (Lindhard) ionizations are closely related but take place on different timescales.

So far, the Migdal effect and other inelastic processes have primarily been studied in atomic targets. As applied to dark matter direct detection, the most complete derivation of the Migdal effect can be found in Ref. [3], while additional discussion can be found in Refs. [10-18]. The effect has been applied to set strong experimental limits in noble liquid targets [17-20]. Charge thresholds in low-threshold semiconductor experiments are even lower [21-23], which makes them even better suited to exploit the Migdal effect. However, inelastic processes are less wellstudied in crystal targets, in part due to the more complicated spectrum of excitations. Moreover, the existing calculation for atomic targets [3] relies on boosting the system to the rest frame of the recoiling nucleus. While convenient for atomic calculations, this method breaks down for semiconductors, as the rest frame of the crystal is a preferred frame. So far, Refs. [15-17] have presented estimates of the Migdal effect in solid state targets, but as of now a fully robust, first principles derivation is still lacking.

In this Letter, we show that the Migdal effect in semiconductors can be described by the $2 \rightarrow 3$ process of DMnucleus scattering in association with a nucleus-electron Coulomb interaction. Furthermore, the details of the electronic band structure can be packaged into the wellstudied energy loss function $\operatorname{Im}(-1 / \epsilon)$, where $\epsilon$ is the dielectric function of the material. When the energy deposited into electronic excitations is close to the plasma frequency, plasmons are resonantly excited. (A plasmon resonance can be thought of as a collective electron excitation or as a longitudinal in-medium photon mode.) A first calculation of the rate for inelastic DM-nucleus scattering with associated plasmon production was 
presented in Ref. [6]. By directly calculating the nonresonant contributions, we show that plasmon production is simply the resonant component of the Migdal effect.

We will begin with a general description of the process in semiconductors and lay out the assumptions in our calculation. We present our main results of the Migdal rate in semiconductors and then clarify its relation to the atomic Migdal effect. Finally, we present sensitivity estimates for experiments using $\mathrm{Si}$ and Ge targets. Detailed calculations are provided in the Supplemental Material (SM) [24].

Description of process.-For an elastic recoil off a free nucleus of mass $m_{N}$, the typical momentum deposited by sub-GeV $\mathrm{DM}$ is $q_{N} \approx m_{\chi} v_{\chi} \approx \mathrm{MeV} \times\left(m_{\chi} / \mathrm{GeV}\right)$, and the typical recoil energy is $E_{N} \approx m_{\chi}^{2} v_{\chi}^{2} / m_{N} \approx$ $35 \mathrm{eV} \times\left(m_{\chi} / \mathrm{GeV}\right)^{2}$, taking the example of an Si target. For sub-GeV DM, the energy and momentum scales are then comparable to various scales inherent to the crystal. Some care is therefore needed with respect to the regime of validity of our approximations.

Concretely, in a typical crystal, each nucleus sits in an approximately harmonic potential with size of $\sim \AA$ and frequency $\bar{\omega} \approx 30-50 \mathrm{meV}$ (see Fig. 1). We will deal with $m_{\chi} \gg 10 \mathrm{MeV}$ such that the inverse momentum transfer $1 / q_{N} \ll \AA$. Then we can consider the interaction of the DM with a single nucleus, the so-called "incoherent approximation" [25]. We will thus compute DM scattering off a nucleus in the ground state of the potential with associated nucleus-electron interaction.

To treat the excited states of the nucleus, we will rely on the "impulse approximation," which is valid if the collision happens quickly relative to the timescale set by the potential well $1 / \bar{\omega}$. (See, e.g., [33].) The initial DMnucleus collision and the emission of the Migdal electrons take place on the timescale $\sim 1 / E_{N}$, during which the nucleus remains near the minimum of the potential well (see Fig. 1). Only at a much later time, $1 / \bar{\omega}$, does the nucleus reach the edge of the unit cell and lose its residual kinetic energy to phonons or become unbound, depending on the initial collision energy. The impulse approximation

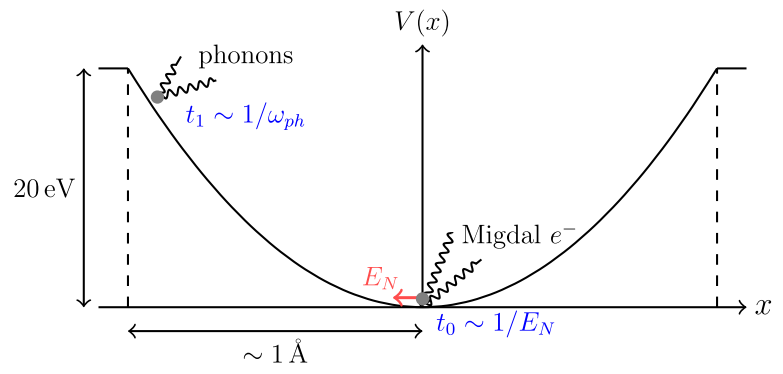

FIG. 1. We compute DM scattering off a nucleus in a harmonic crystal using the impulse approximation, which is valid when the timescale of the initial collision $\left(t_{0}\right)$ is short compared to the timescale to traverse its potential well $\left(t_{1}\right)$, set by the phonon frequency $1 / \bar{\omega}$. This holds for $m_{\chi} \gtrsim 70 \mathrm{MeV}$. thus allows us to model the excited states as plane waves for the duration of the hard collision [34]. In the SM, we show this by calculating the all-orders multiphonon response of a harmonic crystal and find that the approximation holds if the momentum transfer satisfies $q_{N} \gtrsim \sqrt{2 m_{N} \bar{\omega}}$. For DM with the standard velocity profile, this implies $m_{\chi} \gtrsim 70 \mathrm{MeV}$. The incoherent approximation is then satisfied automatically as well. In practice, we will apply a set of cuts to exclude regions of the phase space where the approximations start to break down.

In this discussion, we have focused on the DM momentum transfer to the nucleus $q_{N}$ because the momentum deposited in electrons will be much smaller. The Migdal rate then factorizes as follows:

$$
\frac{d \sigma_{\mathrm{ion}}}{d E_{N} d \omega} \approx \frac{d \sigma_{\mathrm{el}}}{d E_{N}} \frac{d P\left(E_{N}\right)}{d \omega},
$$

where $\omega$ is the energy deposited into electronic excitations, $d \sigma_{\mathrm{el}} / d E_{N}$ is the elastic DM-nucleus cross section, and $d P\left(E_{N}\right) / d \omega$ is the differential ionization probability. This is identical to the expansion made in the bremsstrahlung of a soft photon from a heavy charged particle, and we refer to it as the "soft limit." The soft limit holds as long as $\mid \mathbf{q}_{N}$. $\mathbf{k} \mid \ll m_{N} \omega$ and $k \ll q_{N}$. Estimating $q_{N} \sim v m_{\chi}$ and $k \sim 1-10 \mathrm{keV}$, this translates to $50 \mathrm{MeV} \lesssim m_{\chi} \lesssim 1 \mathrm{GeV}$ and $\omega \gtrsim \mathrm{eV}$, which covers the most relevant parameter space. While our formal result does not rely on the soft limit, it is a useful technical and conceptual simplification when performing the phase space integrals and is valid whenever the impulse approximation holds.

Finally, we will treat the nuclei and tightly bound core electrons together as a particle with charge $Z_{\text {ion }}$ and only consider excitations of the valence electrons. In other words, we assume an ion potential that behaves as $Z_{\text {ion }} e / r$ for large $r$ compared to the wave functions of the inner shell electrons. To account for the effective ion charge at shorter distances, we include a momentumdependent $Z_{\text {ion }}(k)$ in the Fourier-transformed potential, which we obtain using tabulated ionic form factors [35]. While the electron-ion momentum exchange will be $\lesssim 10 \mathrm{keV}$ such that the long-range behavior of the potential is most important, including $Z_{\text {ion }}(k)$ leads to $O(1)$ rate increases.

Calculation.-The Migdal rate is given by the leading order expansion in both the DM-nucleus and the electromagnetic interactions, analogous to bremsstrahlung. We assume a contact interaction between the DM and the nuclei given by the Hamiltonian $H_{\chi}=\left(2 \pi b_{\chi} / m_{\chi}\right) \delta\left(\mathbf{r}_{\chi}-\mathbf{r}_{N}\right)$ for $m_{\chi} \ll m_{N}$, with $b_{\chi}$ the DM-nucleus scattering length and $\mathbf{r}_{\chi}, \mathbf{r}_{N}$ the position operators for the DM and nucleus. (The DM-nucleus elastic cross section is therefore given by $\sigma_{N}=4 \pi b_{\chi}^{2}=A^{2} \sigma_{n}$, with $A$ and $\sigma_{n}$ being, respectively, the atomic mass number and the DM-nucleon elastic cross section.) The electron-nucleus interaction is 
$H_{e}=\int d^{3} \mathbf{r}^{\prime} \epsilon^{-1}\left(\mathbf{r}^{\prime}, \mathbf{r}, \omega\right) Z_{\text {ion }} \alpha /\left|\mathbf{r}^{\prime}-\mathbf{r}_{N}\right|$, with $\mathbf{r}$ the position operator for the electron. $\alpha$ is the electromagnetic fine structure constant, and $\epsilon$ is the frequency-dependent, microscopic dielectric function that encodes the screening by the spectator valence electrons.

In a crystal, the linear response depends on both $\mathbf{r}^{\prime}$ and $\mathbf{r}$ up to the lattice periodicity. The Fourier transform of the response $\epsilon^{-1}\left(\mathbf{r}^{\prime}, \mathbf{r}, \omega\right)$ can then be written as $\epsilon_{\mathbf{K K}^{\prime}}^{-1}(\mathbf{k}, \omega) \equiv \epsilon^{-1}\left(\mathbf{k}+\mathbf{K}, \mathbf{k}+\mathbf{K}^{\prime}, \omega\right)$, where $\mathbf{k}$ is in the first Brillouin zone and $\mathbf{K}, \mathbf{K}^{\prime}$ are reciprocal lattice vectors. $\epsilon_{\mathbf{K K}^{\prime}}^{-1}$ can be regarded as a matrix in the reciprocal lattice vectors, but for $\mathrm{Si}$ and $\mathrm{Ge}$ we find the contribution of the off-diagonal pieces to be subleading. Here, we just present results assuming a diagonal response matrix $\epsilon_{\mathbf{K K}}^{-1}$ and provide the general result in the SM. Including the momentum-dependent ion charge, $H_{e}$ can then be written as
$H_{e}=-4 \pi \alpha \sum_{\mathbf{K}} \int \frac{d^{3} \mathbf{k}}{(2 \pi)^{3}} \frac{Z_{\text {ion }}(|\mathbf{k}+\mathbf{K}|) e^{i\left(\mathbf{r}-\mathbf{r}_{N}\right) \cdot(\mathbf{k}+\mathbf{K})}}{\epsilon_{\mathbf{K} \mathbf{K}}(\mathbf{k}, \omega)|\mathbf{k}+\mathbf{K}|^{2}}$.

We can apply Fermi's golden rule with second-order perturbation theory to compute the cross section for DMnucleus inelastic scattering. We take the initial ions to be in a ground state of a harmonic crystal potential. Following the impulse approximation, we use plane waves for the intermediate and final states. Meanwhile, the electron states are treated as Bloch states. Though the computation itself is a straightforward application of second-order perturbation theory, the formulas and derivation are somewhat lengthy due to the appearance of the reciprocal lattice vectors and a form factor for the recoiling ion. We refer the reader to the SM for further details and only present the final result here:

$$
\begin{aligned}
& \frac{d \sigma}{d \omega}=\frac{2 \pi^{2} A^{2} \sigma_{n}}{m_{\chi}^{2} v_{\chi}} \int \frac{d^{3} \mathbf{q}_{N}}{(2 \pi)^{3}} \int \frac{d^{3} \mathbf{p}_{f}}{(2 \pi)^{3}} \delta\left(E_{i}-E_{f}-\omega-E_{N}\right) \times F\left(\mathbf{p}_{i}-\mathbf{p}_{f}-\mathbf{q}_{N}-\mathbf{k}-\mathbf{K}\right)^{2} \times \sum_{\mathbf{K}} \int \frac{d^{3} \mathbf{k}}{(2 \pi)^{3}} \frac{4 \alpha Z_{\mathrm{ion}}^{2}(|\mathbf{k}+\mathbf{K}|)}{\left|\epsilon_{\mathbf{K K}}(\mathbf{k}, \omega)\right|^{2}} \\
& \times\left[\frac{1}{\omega-\mathbf{q}_{N} \cdot(\mathbf{k}+\mathbf{K}) / m_{N}}-\frac{1}{\omega}\right]^{2} \underbrace{\frac{4 \pi^{2} \alpha}{V} \sum_{\mathbf{p}_{e}} \frac{\left|\left[\mathbf{p}_{e}+\mathbf{k}\left|e^{i \mathbf{r} \cdot \mathbf{K}}\right| \mathbf{p}_{e}\right]_{\Omega}\right|^{2}}{|\mathbf{k}+\mathbf{K}|^{2}}\left[f\left(\mathbf{p}_{e}\right)-f\left(\mathbf{p}_{e}+\mathbf{k}\right)\right] \delta\left(\omega_{\mathbf{p}_{e}+\mathbf{k}}-\omega_{\mathbf{p}_{e}}-\omega\right)}_{\operatorname{Im}\left[\epsilon_{\mathbf{K K}}(\mathbf{k}, \omega)\right]},
\end{aligned}
$$

where $\mathbf{q}_{N}$ and $\mathbf{p}_{f}$ are the final ion and DM momentum, respectively, and $\mathbf{k}+\mathbf{K}$ is the momentum deposited to the electrons. In the first part of Eq. (3), we see the same factors and phase space integral that appear for elastic DM-nucleus scattering except for the additional energy $\omega$ being deposited in electronic excitation. While for free nucleus scattering there would be a momentum conservation delta function, here we have a form factor $F$ that encodes the details of the ion ground state, and, for a harmonic crystal, this is given by

$F\left(\mathbf{p}_{i}-\mathbf{p}_{f}-\mathbf{q}\right) \equiv\left(\frac{4 \pi}{m_{N} \bar{\omega}}\right)^{3 / 4} e^{\left[\left(-\left|\mathbf{p}_{i}-\mathbf{p}_{f}-\mathbf{q}\right|^{2}\right) /\left(2 m_{N} \bar{\omega}\right)\right]}$,

where $\bar{\omega}$ is an oscillator frequency averaged with respect to the density of states $D(\omega)$ and the thermal Bose factor. The remaining pieces of Eq. (3) contain the probability for exciting the electron. We sum over all initial and final electron states $\mathbf{p}_{e}$ and $\mathbf{p}_{e}+\mathbf{k}$, weighted by the occupation numbers $f$, and where band indices have been suppressed. The electronic wave function overlaps $\left[\mathbf{p}_{e}+\mathbf{k}\left|e^{i \mathbf{r} \cdot \mathbf{K}}\right| \mathbf{p}_{e}\right]_{\Omega}$ are performed over the unit cell, and $V$ is the volume of the crystal.

In Eq. (3), the bracketed quantity can be rewritten in terms of the imaginary part of the dielectric function in the random phase approximation $\operatorname{Im}\left[\epsilon_{\mathbf{K} \mathbf{K}}(\mathbf{k}, \omega)\right]$. Then we can write $\operatorname{Im}\left[\epsilon_{\mathbf{K K}}(\mathbf{k}, \omega)\right] /\left|\epsilon_{\mathbf{K K}}(\mathbf{k}, \omega)\right|^{2}=\operatorname{Im}\left[-1 / \epsilon_{\mathbf{K K}}(\mathbf{k}, \omega)\right]$, which is the energy loss function (ELF) governing the energy loss of charged particles in a material. Physically, the ion-electron interaction in the inelastic process can be encapsulated in the same ELF as ions passing through a material. Since the ELF is a well-measured and calculated quantity in many materials, this provides a useful starting point for numerical evaluations of Eq. (3).

In the soft limit $|\mathbf{k}+\mathbf{K}| \ll\left|\mathbf{q}_{N}\right|$, the cross section factorizes as in Eq. (1), and the form factor $F$ only modifies the elastic recoil cross section. Then the differential ionization probability is

$$
\begin{aligned}
\frac{d P}{d \omega}= & \frac{(4 \pi \alpha)^{2}}{\omega^{4}} \sum_{\mathbf{p}_{e}} \int \frac{d^{3} \mathbf{k}}{(2 \pi)^{3}} Z_{\mathrm{ion}}^{2}(k) \frac{\left|\mathbf{v}_{N} \cdot \mathbf{k}\right|^{2}}{k^{4}} \frac{\left|\left[\mathbf{p}_{e}+\mathbf{k} \mid \mathbf{p}_{e}\right]_{\Omega}\right|^{2}}{V|\epsilon(\mathbf{k}, \omega)|^{2}} \\
& \times\left[f\left(\mathbf{p}_{e}\right)-f\left(\mathbf{p}_{e}+\mathbf{k}\right)\right] \delta\left(\omega_{\mathbf{p}_{e}+\mathbf{k}}-\omega_{\mathbf{p}_{e}}-\omega\right) \\
= & 4 \alpha \int \frac{d^{3} \mathbf{k}}{(2 \pi)^{3}} \frac{Z_{\mathrm{ion}}^{2}(k)}{\omega^{4}} \frac{\left|\mathbf{v}_{N} \cdot \mathbf{k}\right|^{2}}{k^{2}} \operatorname{Im}\left[\frac{-1}{\epsilon(\mathbf{k}, \omega)}\right]
\end{aligned}
$$

with $\mathbf{v}_{N} \equiv \mathbf{q}_{N} / m_{N}$. This simplified formula is only valid for $\mathbf{k}$ in the first Brillouin zone, while we used the full expressions used in our numerical results. Equation (6) was also derived in [6], but that work did not account for the ion ground state or electron momentum transfers outside of the first Brillouin zone since it was focused on long-wavelength plasmons. Furthermore, [6] used an 
analytic approximation for $\epsilon(\mathbf{k}, \omega)$ near the plasmon pole. In the results below, we will study the impact of accounting for the ion ground state and use numerical calculations of $\epsilon(\mathbf{k}, \omega)$ that are valid away from the plasmon resonance. Before doing so, we clarify the relation of this process to the atomic Migdal effect.

Comparison with atomic Migdal effect.-In Migdal's original calculation $[7,8]$ for an atomic target, the ground state of the electron cloud $(|i\rangle)$ is first boosted to the rest frame of the moving nucleus $|i\rangle \rightarrow e^{i m_{e} \mathbf{v}_{N} \cdot \sum_{\beta} \mathbf{r}_{\beta}}|i\rangle$. He then computes the overlap with the excited states $\langle f|$ :

$$
\mathcal{M}_{i f}=\left\langle f\left|e^{i m_{e} \mathbf{v}_{N} \cdot \sum_{\beta} \mathbf{r}_{\beta}}\right| i\right\rangle \approx i m_{e}\left\langle f\left|\mathbf{v}_{N} \cdot \sum_{\beta} \mathbf{r}_{\beta}\right| i\right\rangle,
$$

where $\beta$ runs over all the electrons in the atom. The transition probabilities $\left|\mathcal{M}_{\text {if }}\right|^{2}$ can then be evaluated with known atomic wave functions, and it was found that single ionizations dominate for sub-GeV dark matter [3].

To demonstrate the connection to the semiconductor Migdal effect derived above, we instead rewrite Eq. (7) using the following operator identity: $\left\langle f\left|\sum_{\beta} \mathbf{r}_{\beta}\right| i\right\rangle=$ $-i\left\langle f\left|\sum_{\beta} \mathbf{p}_{\beta}\right| i\right\rangle / m_{e} \omega=i\left\langle f\left|\sum_{\beta}\left[\mathbf{p}_{\beta}, H_{0}\right]\right| i\right\rangle / m_{e} \omega^{2}$, where again $\omega=E_{f}-E_{i}$ is the total energy deposited and $H_{0}$ the electron Hamiltonian. We assume a nonrelativistic [36] Hamiltonian such that the $H_{0}$ is a sum of kinetic terms, the Coulomb interaction terms between electrons, and the Coulomb interaction of the electrons with the nucleus. Then the commutator $\sum_{\beta}\left[\mathbf{p}_{\beta}, H_{0}\right]$ will be proportional to the total force from the nucleus since the electron-electron forces cancel out. Contracting the matrix element with $\mathbf{v}_{N}$, we find (see also [37])

$$
\left\langle f\left|\sum_{\beta} \mathbf{v}_{N} \cdot \mathbf{r}_{\beta}\right| i\right\rangle=\frac{1}{m_{e} \omega^{2}}\left\langle f\left|\sum_{\beta} \frac{Z_{N} \alpha \mathbf{v}_{N} \cdot \hat{\mathbf{r}}_{\beta}}{\left|\mathbf{r}_{\beta}-\mathbf{r}_{N}\right|^{2}}\right| i\right\rangle,
$$

with $\mathbf{r}_{N}$ the position operator of the nucleus. In the rhs matrix element above, we see the time derivative of the dipole potential from a nucleus that has been displaced by $\left|\mathbf{r}_{N}\right| \ll\left|\mathbf{r}_{\beta}\right|$. This is already very similar to the Coulomb potential in Eq. (2) and suggestive of the same physical interpretation as in the semiconductor case. One can now take the Fourier transform and evaluate the transition probability for single ionizations:

$$
\begin{aligned}
\frac{d P\left(E_{N}\right)}{d \omega} \approx & \left(\frac{4 \pi Z_{N} \alpha}{\omega^{2}}\right)^{2} \sum_{i, f}\left|\int \frac{d^{3} \mathbf{k}}{(2 \pi)^{3}} \frac{\mathbf{v}_{N} \cdot \mathbf{k}}{k^{2}}\left\langle f\left|e^{i \mathbf{k} \cdot \mathbf{r}}\right| i\right\rangle\right|^{2} \\
& \times \delta\left(E_{i}+\omega-E_{f}\right),
\end{aligned}
$$

where we have dropped the $e^{-i \mathbf{k} \cdot \mathbf{r}_{N}}$ phase factor in the soft limit. We have thus shown that the atomic Migdal effect has a form nearly identical to Eq. (5) for semiconductors, up to a few differences reflecting the different physical systems.
In Eq. (5), the integral over $\mathbf{k}$ appears outside the amplitude squared; this reflects conservation of crystal momentum in the semiconductor, which requires that the final state have momentum $\mathbf{p}_{e}+\mathbf{k}$, whereas the atomic states are not momentum eigenstates. The nucleus charge $Z_{N}$ appears here since we are considering the all-electron wave functions, whereas in the semiconductor case we effectively integrated out the core electrons and treated the ion with effective charge $Z_{\text {ion }}(k)$. Finally, in Eq. (5), we accounted for the in-medium dielectric screening $1 /|\epsilon(\mathbf{k}, \omega)|^{2}$ due to all the other electrons, which is neglected in the atomic case.

With this result, we find an equivalent formulation of the atomic Migdal effect, Eq. (9), but which has a physical interpretation that applies also in semiconductors. Previously, Ref. [38] assumed the atomic formulation in Eq. (7) could be generalized directly to semiconductors. However, the boosting argument used to obtain Eq. (7) does not apply in this case, since in a crystal there is a preferred coordinate frame. In other words, applying a boost operator would boost all nuclei in the lattice. Specifically, the two approaches are not equivalent because the operator relation, Eq. (8), only applies for an individual atom: in the presence of a lattice of nuclei, we would have contributions from all nuclei on the rhs of Eq. (8). Reference [15] attempted to address this subtlety by using atom-centered localized Wannier functions in Eq. (7) to mitigate the contribution from the remaining nuclei in the crystal. For $\mathrm{Si}$ and $\mathrm{Ge}$ in particular, the Wannier approach is found, however, to be computationally challenging due to slow numerical convergence [15].

Between these different starting points, Eq. (9) has a clear physical interpretation as the in-medium analog of bremsstrahlung, which nicely generalizes to the semiconductor case. This interpretation is discussed further in the SM. The final result in semiconductors can moreover be expressed in terms of the ELF, which can be calculated with a number of public codes. We therefore argue for this approach in generalizing the atomic Migdal effect.

Results. - The Migdal rate is given by $d R / d \omega=$ $N_{T} n_{\chi} \int d^{3} \mathbf{v}_{\chi} f\left(\mathbf{v}_{\chi}\right) v_{\chi} d \sigma / d \omega$, where $N_{T}$ is number of target nuclei per kilogram and $n_{\chi}=\rho_{\chi} / m_{\chi}$ is the DM number density. We take $\rho_{\chi}=0.4 \mathrm{GeV} / \mathrm{cm}^{3}$ and assume the standard halo model for the DM velocity distribution $f(\mathbf{v})$ with escape velocity $v_{\text {esc }}=500 \mathrm{~km} / \mathrm{s}[39,40]$ and Earth velocity $v_{e}=240 \mathrm{~km} / \mathrm{s}$. We calculate the dielectric function with the public code GPAW [41-44]. The wave functions are computed on an $8 \times 8 \times 8 k$-space grid for $\mathrm{Si}$ and a $12 \times 12 \times 12$ grid for Ge. The TB09 exchangecorrelation functional [45] is used and local field effects are incorporated [46]. A scissor correction is applied to match on to the experimentally measured band gap in both materials. We have averaged the ELF over crystal directions, and for computational reasons, we currently only include the diagonal components of the loss function, 

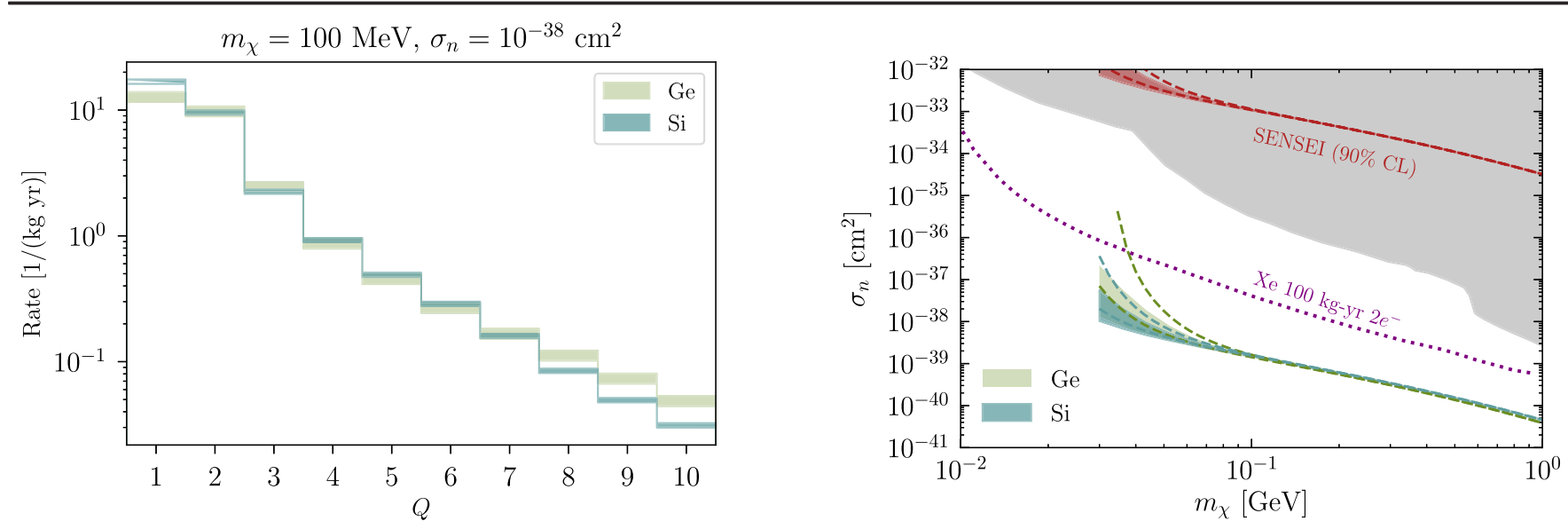

FIG. 2. (Left) Electron recoil spectrum in $\mathrm{Si}$ and Ge from the Migdal effect in semiconductors, assuming DM-nucleon cross section of $10^{-38} \mathrm{~cm}^{2}$ and DM mass $100 \mathrm{MeV}$. The differential rate is translated into total number of electron-hole pairs created $(Q)$ using Eq. (5.1) of Ref. [48]. (Right) Expected 90\% CL sensitivity to DM-nucleon cross section $\sigma_{n}$ assuming a heavy mediator and $1 \mathrm{~kg}$-year of exposure. We take $Q \geq 2$ and zero background, corresponding to an upper limit of 2.4 events. The red line is a 90\% CL limit obtained using the recent upper limit on the 2-electron rate from SENSEI [22], while the shaded region includes bounds from XENON1T [20], LUX [19], CRESST III [49], and CDEX [50], as well as a recast of XENON10 [51], XENON100 [52], and XENON1T [53] data in terms of the Migdal effect [17]. For comparison, we show a projection for the Migdal effect in xenon (dotted line) based on the atomic ionization signal [17]. In both panels, the shaded bands are an estimate of the theoretical uncertainty due to the impulse approximation, obtained by varying the threshold on $E_{N}$ from $4 \bar{\omega}$ to $9 \bar{\omega}$.

although we verified that the off-diagonal terms do not contribute more than an $\mathcal{O}(1)$ amount to the total rate. Further details, more refined numerical studies, and other applications will be presented in a forthcoming publication [47].

The resulting rates and sensitivity estimates are shown in Fig. 2 for $\mathrm{Si}$ and Ge. The bands indicate an estimate of the theory uncertainty due the breakdown of the impulse approximation for low energy nuclear recoils. We find that our calculation starts to break down for $m_{\chi} \lesssim 50 \mathrm{MeV}$, at which point one has to go beyond the impulse approximation by matching onto the phonon regime. The dashed lines in the right-hand panel indicate the free ion approximation, where the $F$ form factor is replaced with a $\delta$ function. We find that the free ion approximation is excellent in the regime where the impulse approximation applies, further validating the approach in [6]. Compared to [6], we find, however, significantly stronger sensitivity by including the contributions away from the plasmon resonance. Most importantly, we confirm that the Migdal rate for a low threshold detector such as SENSEI, DAMIC, or SuperCDMS is much higher than in noble liquid detectors. This is due to the lower ionization gap, the $\omega^{-4}$ scaling in Eq. (6), and the possibility of detecting all electronic excitations rather than only atomic ionizations.

We are grateful to Noah Kurinsky, Daniel Baxter, Gordan Krnjaic, Toby Opferkuch, and Chih-Pan Wu for useful discussions and to Yonatan Kahn for useful discussions and valuable comments on the draft. We further thank Diego Redigolo for useful discussions, proofreading the manuscript, and collaboration on related work. T. L. and
J. K. are supported by the Department of Energy under Grant No. DE-SC0019195 and a UC Hellman fellowship. T. L. is also supported by an Alfred P. Sloan Foundation fellowship.

Note added.-In the final stages of preparing this work, [54] appeared, which studies the multiphonon response and has some overlap with the calculation in our Supplemental Material.

* Corresponding author. simon.knapen@cern.ch Corresponding author. jkozaczuk@physics.ucsd.edu ${ }^{\ddagger}$ Corresponding author. tongyan@physics.ucsd.edu

[1] M. Battaglieri et al., arXiv:1707.04591.

[2] R. Kolb et al., Basic research needs for dark matter small projects new initiatives, Technical Report, DoE, 2018, https://www.osti.gov/servlets/purl/1659757.

[3] M. Ibe, W. Nakano, Y. Shoji, and K. Suzuki, J. High Energy Phys. 03 (2018) 194.

[4] C. Kouvaris and J. Pradler, Phys. Rev. Lett. 118, 031803 (2017).

[5] N. Kurinsky, D. Baxter, Y. Kahn, and G. Krnjaic, Phys. Rev. D 102, 015017 (2020).

[6] J. Kozaczuk and T. Lin, Phys. Rev. D 101, 123012 (2020).

[7] A. Migdal, Sov. Phys. JETP 9, 1163 (1939).

[8] A. B. Migdal, Qualitative Methods in Quantum Theory (W.A. Benjamin, Inc., Reading, 1977), Vol. 48.

[9] J. Lindhard, V. Nielsen, M. Scharff, and P. Thomsen, Kgl. Danske Videnskab., Selskab. Mat. Fys. Medd. 33, 1 (1963), 
http://gymarkiv.sdu.dk/MFM/kdvs/mfm\%2030-39/mfm33-10.pdf.

[10] R. Bernabei et al., Int. J. Mod. Phys. A 22, 3155 (2007).

[11] N. F. Bell, J. B. Dent, J. L. Newstead, S. Sabharwal, and T. J. Weiler, Phys. Rev. D 101, 015012 (2020).

[12] D. Baxter, Y. Kahn, and G. Krnjaic, Phys. Rev. D 101, 076014 (2020).

[13] G. Grilli di Cortona, A. Messina, and S. Piacentini, J. High Energy Phys. 11 (2020) 034.

[14] K. D. Nakamura, K. Miuchi, S. Kazama, Y. Shoji, M. Ibe, and W. Nakano, Prog. Theor. Exp. Phys. 2021, 013 C01 (2021).

[15] Z.-L. Liang, L. Zhang, F. Zheng, and P. Zhang, Phys. Rev. D 102, 043007 (2020).

[16] C. P. Liu, C.-P. Wu, H.-C. Chi, and J.-W. Chen, Phys. Rev. D 102, 121303(R) (2020).

[17] R. Essig, J. Pradler, M. Sholapurkar, and T.-T. Yu, Phys. Rev. Lett. 124, 021801 (2020).

[18] M. J. Dolan, F. Kahlhoefer, and C. McCabe, Phys. Rev. Lett. 121, 101801 (2018).

[19] D. Akerib et al. (LUX Collaboration), Phys. Rev. Lett. 122, 131301 (2019).

[20] E. Aprile et al. (XENON Collaboration), Phys. Rev. Lett. 123, 241803 (2019).

[21] A. Aguilar-Arevalo et al. (DAMIC Collaboration), Phys. Rev. Lett. 123, 181802 (2019).

[22] L. Barak et al. (SENSEI Collaboration), Phys. Rev. Lett. 125, 171802 (2020).

[23] D. Amaral et al. (SuperCDMS Collaboration), Phys. Rev. D 102, 091101 (2020).

[24] See Supplemental Material at http://link.aps.org/ supplemental/10.1103/PhysRevLett.127.081805 for the complete derivation of the Migdal effect, discussion of the impulse approximation used for multiphonon production, details of the rate calculation, and an alternative semiclassical derivation.

[25] Coherent scattering with multiple nuclei (that is, phonon excitation) occurs for $m_{\chi} \lesssim \mathrm{MeV}$ and was treated in Refs. [26-32].

[26] S. Knapen, T. Lin, M. Pyle, and K. M. Zurek, Phys. Lett. B 785, 386 (2018).

[27] S. Griffin, S. Knapen, T. Lin, and K. M. Zurek, Phys. Rev. D 98, 115034 (2018).

[28] S. M. Griffin, K. Inzani, T. Trickle, Z. Zhang, and K. M. Zurek, Phys. Rev. D 101, 055004 (2020).

[29] T. Trickle, Z. Zhang, K. M. Zurek, K. Inzani, and S. Griffin, J. High Energy Phys. 03 (2020) 036.

[30] P. Cox, T. Melia, and S. Rajendran, Phys. Rev. D 100, 055011 (2019).

[31] B. Campbell-Deem, P. Cox, S. Knapen, T. Lin, and T. Melia, Phys. Rev. D 101, 036006 (2020); 102, 019904(E) (2020).

[32] S. M. Griffin, Y. Hochberg, K. Inzani, N. Kurinsky, T. Lin, and T. C. Yu, Phys. Rev. D 103, 075002 (2021).

[33] G. I. Watson, J. Phys. Condens. Matter 8, 5955 (1996).

[34] In the atomic Migdal effect, the recoiling ion is often modeled as a semiclassical current. In this context, the "impulse approximation" corresponds to a stronger set of assumptions than those employed here.

[35] P. J. Brown, A. G. Fox, E. N. Maslen, M. A. OKeefe, and B. T. M. Willis, Intensity of diffracted intensities, in International Tables for Crystallography (American Cancer
Society, 2006), Chap. 6.1, pp. 554-595, https://onlinelibrary .wiley.com/doi/pdf/10.1107/97809553602060000600.

[36] Relativistic corrections can be important for inner shell electrons, but the rate is dominated by the nonrelativistic outer shells.

[37] F. Rösel, D. Trautmann, and G. Baur, Nucl. Instrum. Methods Phys. Res. 192, 43 (1982).

[38] R. Essig, J. Perez-Ros, H. Ramani, and O. Slone, Phys. Rev. Research 1, 033105 (2019).

[39] T. Piffl et al., Astron. Astrophys. 562, A91 (2014).

[40] G. Monari, B. Famaey, I. Carrillo, T. Piffl, M. Steinmetz, R. F. G. Wyse, F. Anders, C. Chiappini, and K. Janen, Astron. Astrophys. 616, L9 (2018).

[41] J. J. Mortensen, L. B. Hansen, and K. W. Jacobsen, Phys. Rev. B 71, 035109 (2005).

[42] J. Enkovaara et al., J. Phys. Condens. Matter 22, 253202 (2010).

[43] M. Walter, H. Häkkinen, L. Lehtovaara, M. Puska, J. Enkovaara, C. Rostgaard, and J. J. Mortensen, J. Chem. Phys. 128, 244101 (2008).

[44] J. Yan, J. J. Mortensen, K. W. Jacobsen, and K. S. Thygesen, Phys. Rev. B 83, 245122 (2011).

[45] F. Tran and P. Blaha, Phys. Rev. Lett. 102, 226401 (2009).

[46] S. L. Adler, Phys. Rev. 126, 413 (1962).

[47] S. Knapen, J. Kozaczuk, and T. Lin, arXiv:2101.08275 [Phys. Rev. D (to be published)].

[48] R. Essig, M. Fernandez-Serra, J. Mardon, A. Soto, T. Volansky, and T.-T. Yu, J. High Energy Phys. 05 (2016) 046.

[49] A. H. Abdelhameed et al. (CRESST Collaboration), Phys. Rev. D 100, 102002 (2019).

[50] Z. Z. Liu et al. (CDEX Collaboration), Phys. Rev. Lett. 123, 161301 (2019).

[51] J. Angle et al. (XENON10 Collaboration), Phys. Rev. Lett. 107, 051301 (2011); 110, 249901(E) (2013).

[52] E. Aprile et al. (XENON Collaboration), Phys. Rev. D 94, 092001 (2016); 95, 059901(E) (2017).

[53] E. Aprile et al. (XENON Collaboration), Phys. Rev. Lett. 123, 251801 (2019).

[54] Y. Kahn, G. Krnjaic, and B. Mandava, preceding Letter, Phys. Rev. Lett. 127, 081804 (2021).

[55] J. Lindhard, Kgl. Danske Videnskab. Selskab Mat.-fys. Medd. 28, 1 (1954), http://gymarkiv.sdu.dk/MFM/kdvs/ mfm\%2020-29/mfm-28-8.pdf.

[56] S. L. Adler, Phys. Rev. 126, 413 (1962).

[57] J. Deslippe, G. Samsonidze, D. A. Strubbe, M. Jain, M. L. Cohen, and S. G. Louie, Comput. Phys. Commun. 183, 1269 (2012).

[58] N. D. Mermin, Phys. Rev. B 1, 2362 (1970).

[59] M. Vos and P. L. Grande, J. Phys. Chem. Solids Suppl. 124, 242 (2019).

[60] M. J. Weber, Handbook of Optical Materials (CRC Press, Boca Raton, 2003).

[61] H.-C. Weissker, J. Serrano, S. Huotari, E. Luppi, M. Cazzaniga, F. Bruneval, F. Sottile, G. Monaco, V. Olevano, and L. Reining, Phys. Rev. B 81, 085104 (2010).

[62] J. M. F. Gunn and M. Warner, Z. Phys. B 56, 13 (1984).

[63] H. Schober, J. Neutron Res. 17, 109 (2014).

[64] J. Law, Nucl. Phys. A286, 339 (1977).

[65] J. S. Levinger, Phys. Rev. 90, 11 (1953). 